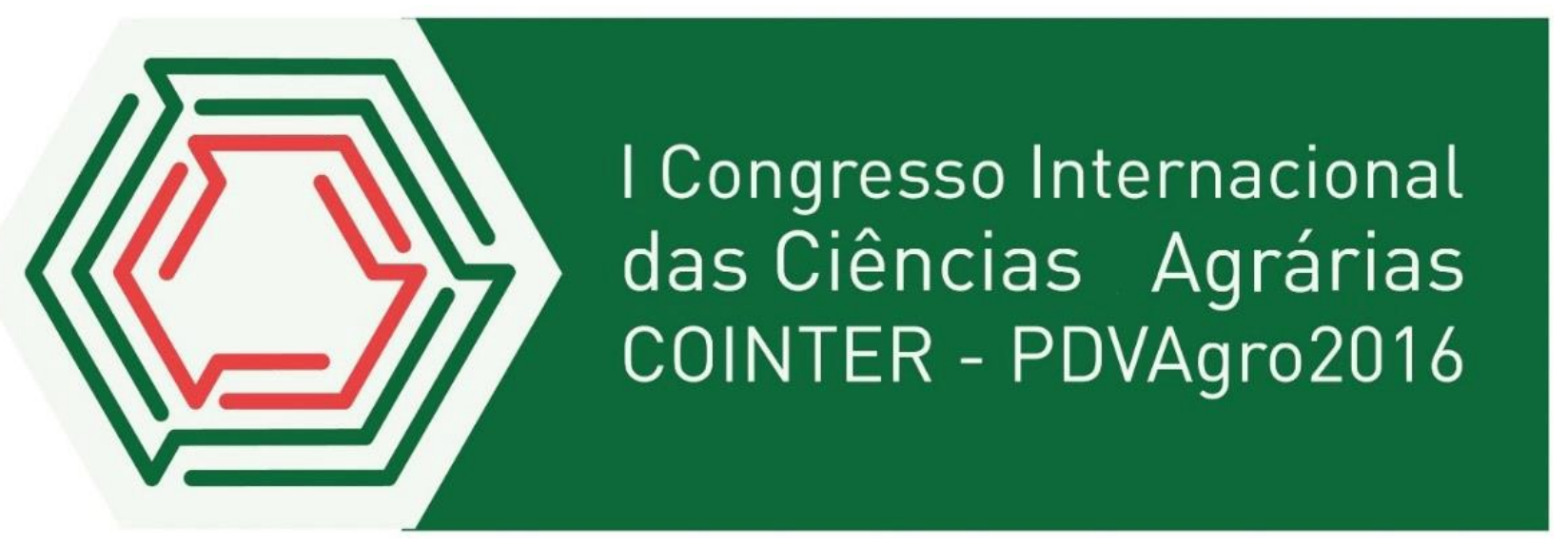

\title{
EFICIÊNCIA DAS PLANTAS DE COBERTURA EM CULTIVOS DE MELÃO NA FIXAÇÃO BIOLÓGICA DE NITROGÊNIO
}

\section{NO SEMIÁRIDO DE PERNAMBUCO}

\author{
Apresentação: Comunicação Oral
}

\begin{abstract}
Gustavo da Costa Lima ${ }^{1}$; Jessyca Adriana Gomes Florêncio da Silva ${ }^{2}$; Renata Janaína Carvalho de Souza $^{3}$; Vanderlise Giongo ${ }^{4}$; Ana Dolores Santiago de Freitas ${ }^{5}$
\end{abstract}

\section{Resumo}

Objetivou-se, com o presente trabalho, verificar o desempenho de plantas de cobertura na fixação biológica de nitrogênio em cultivos de melão no Semiárido de Pernambuco. Os estudos foram conduzidos no Campo Experimental de Bebedouro, propriedade da Embrapa Semiárido, localizado na cidade de Petrolina - PE em plantios de melão irrigados. Os experimentos adotam um delineamento em blocos ao acaso com parcelas subdivididas e quatro repetições. As parcelas correspondem a duas formas de manejo da biomassa (a incorporação da biomassa dos adubos verdes e das plantas espontâneas ao solo ou a deposição da biomassa na superfície do solo) produzida pelos três tratamentos de adubos verdes que constituem as subparcelas. Os tratamentos de adubos verdes corresponderam a duas misturas de espécies (denominadas de coquetéis) e um terceiro tratamento onde é permitido o crescimento de plantas espontâneas. Para a estimativa da 
FBN, foram realizadas coletas do material vegetal dos adubos verdes na época do corte, em quadrados de $0,25 \mathrm{~m}^{2}$, lançados aleatoriamente, nas linhas dos coquetéis vegetais, com três repetições por parcela. Os dados de biomassa extraídos do cultivo dos coquetéis vegetais contribuíram com maior acúmulo de biomassa quando comparado com os tratamentos onde foi permitido o crescimento das espécies espontâneas. Entre as leguminosas, a Mucuna destacou-se na produção de biomassa, variando de 2,143 a 3,619 $\mathrm{t} \mathrm{ha}^{-1}$. Todas as leguminosas utilizadas no experimento apresentam-se como fixadoras de $\mathrm{N}$, por apresentarem valores baixos de $\delta{ }^{15} \mathrm{~N}$, quando comparadas com os valores encontrados pelas plantas referência, diferenças que ultrapassam 2 \%. Mucunas apresentam-se como boa alternativa de cultivo como planta fixadora de $\mathrm{N}$ e fornecedora de biomassa como adubo verde

Palavras-Chave: Adubo verde, leguminosas, fixação biológica de nitrogênio.

\section{Introdução}

O cultivo de melão é comum nos solos do semiárido e é uma fonte de renda bastante importante para pequenos e médios produtores. Apesar do semiárido cobrir a maioria dos estados da Região Nordeste e possuir uma extensão de total de $982.563,3 \mathrm{~km}^{2}$ (IBGE, 2005), o cultivo de meloeiros ocorre em perímetros irrigados e próximos a rios (IBGE, 2013). A região delimitada pelo semiárido nordestino possui características climáticas, pedológicas e biológicas que as definem. Possui precipitações médias anuais iguais ou inferiores 800 mm, insolação média de 2.800 h/ano, temperaturas médias anuais 23 a $27 \mathrm{C}$, regime de chuva marcado pela irregularidade (espaço/tempo), constitui o Bioma Caatinga, há também limitações pluviométricas, solos, de maioria, arenoso-argiloso e pobres em matéria orgânica (SUDENE, 2016).

O uso de plantas de cobertura como adubo verde em consórcio, é uma pratica que melhora as qualidades químicas do solo. A utilização destas e outras técnicas são responsáveis por adicionar matéria orgânica e nitrogênio aos sistemas, devido a capacidade de fixar nitrogênio atmosférico através de associações com micro-organismos diazotróficos (Zotarelli et al., 2012). Utilizar essas espécies pode complementar ou substituir a adubação com fertilizantes nitrogenados, dependentes de energia fóssil para sua fabricação, surgindo assim como uma alternativa sustentável ao meio ambiente. 
A fixação biológica do nitrogênio $(\mathrm{FBN})$, é a principal via de incorporação de nitrogênio atmosférico $\left(\mathrm{N}_{2}\right)$ no solo, sendo responsável por cerca de $65 \%$ da entrada de $\mathrm{N}$ no ecossistema terreno. Estima-se que de 44 a 66 milhões de toneladas métricas de N2 são fixados por leguminosas de importância agrícola anualmente e outros 3 a 5 milhões de toneladas métricas são fixados por leguminosas em ecossistemas naturais (GRAHAM e VANCE, 2003).

Contudo, não existem informações sobre as quantidades de $\mathrm{N}$ que podem ser aportadas pela fixação biológica em adubos verdes na região semiárida do Brasil. Essa informação é importante para o estabelecimento de um manejo adequado de cultivos com adubos verdes, pois subsidia a escolha de espécies com potencial de promover um balanço de nutrientes mais favorável ao sistema.

Dessa forma, o objetivo do trabalho é estimar o potencial de FBN por plantas de cobertura em cultivos de melão no Semiárido de Pernambuco.

\section{Adubação Verde}

A adubação verde tem sido empregada como alternativa ao uso a adubação nitrogenados. Essas plantas quando cultivadas em consórcio (Paulino et al., 2009) ou antecedendo a cultura principal (Ojien et al., 2014), adicionam matéria orgânica e nitrogênio aos sistemas, devido a capacidade de fixar nitrogênio atmosférico através de associações com micro-organismos diazotróficos (Zotarelli et al., 2007). Vários estudos têm sido realizados com a finalidade de avaliar e estimar a contribuição da adubação verde para o solo e as culturas de interesse.

Para a recomendação das espécies a serem utilizadas como adubos verdes em determinada região, deve-se procurar combinações dos fatores que atendam às exigências locais, dando-se preferência àquelas que produzam um maior volume de matéria seca, estejam menos sujeitas a pragas e doenças e que possuam sementes relativamente uniformes e fáceis de semear manualmente ou por meio de máquinas (BARRETO e FERNANDES, 2001).

Sabe-se que a utilização de plantas de cobertura traz diversos benefícios a cultura, além de melhorar as propriedades físicas, químicas e biológicas do solo. As leguminosas e as gramíneas são normalmente as espécies mais empregadas para esse fim, tanto uma quanto a outra trazem melhorias para o sistema solo- planta. A utilização de espécies não leguminosas na adubação verde ajuda a reduzir as perdas de $\mathrm{N}$, através da imobilização temporária do $\mathrm{N}$ em sua biomassa. 
Em solo do Cerrado, Torres et al. (2005) demonstraram que a decomposição e a liberação de nitrogênio de resíduos culturais de plantas de cobertura são bastante variáveis de acordo com a espécie de adubo verde. O milheto apresentou a maior produção de massa seca. Dentre as leguminosas, a maior produção foi da crotalária. As espécies que apresentaram maior velocidade de decomposição foram a crotalária e guandu.

No entanto, autores defendem que os efeitos benéficos apresentados pela adubação verde com leguminosas sao superiores à espécie não leguminosa. Ashraf et al. (2004) analisaram a utilização da sesbânia (Sesbania aculeata (Willd.) Pers.) e do milho (Zea mays L.) como adubos verdes para dois cultivares de arroz e os resultados mostraram que a leguminosa, além de representar uma fonte direta de $\mathrm{N}$ disponível para a planta, também tem grande potencial para aumentar o $\mathrm{N}$ disponível do solo para as culturas e para conservação do teor de $\mathrm{N}$.

Perin et al. (2009) estudaram o efeito da cobertura viva, formada por leguminosas herbáceas perenes (amendoim forrageiro - Arachis pintoi Krapov. \& W.C. Greg., cudzu tropical - Pueraria phaseoloides (Roxb.) Benth., siratro - Macroptilium atropurpureum (Moc. \& Sessé ex DC.) Urb.) sobre a produção de bananeira cultivar Nanicão, no município de Seropédica, Rio de Janeiro. Todas as leguminosas proporcionaram maior crescimento das bananeiras, maior número de folhas emitidas e maior proporção de cachos colhidos, em relação aos tratamentos com vegetação espontânea (com e sem $\mathrm{N}$-fertilizante). Siratro e cudzu tropical promoveram condições adequadas ao desenvolvimento das bananeiras, ocasionando ganhos de produtividade e eliminação da adubação nitrogenada no bananal. O potencial benéfico de tais leguminosas, proporcionando ganhos na produtividade de banana, qualifica essas espécies como alternativas promissoras para a fertilidade do solo e nutrição das bananeiras.

\section{Metodologia}

O trabalho foi conduzido no Campo Experimental de Bebedouro, propriedade da Embrapa Semiárido, localizado na cidade de Petrolina - PE (latitude $09^{\circ} 09^{\prime} \mathrm{S}$, longitude $40^{\circ} 22^{\prime} \mathrm{W}$ e altitude $365,5 \mathrm{~m})$ em plantios de melão irrigado. O solo da área é classificado como Argissolo VermelhoAmarelo distrófico plíntico, textura média/argilosa, relevo plano. O clima da região é enquadrado como BSwh, segundo classificação de Köppen, com temperatura média anual em torno de $26,8{ }^{\circ} \mathrm{C}$, precipitação média anual de 541 mm e vegetação nativa de caatinga hiperxerófila.

Os experimentos adotam um delineamento em blocos ao acaso com parcelas subdivididas e quatro repetições. As parcelas correspondem a duas formas de manejo da biomassa (a incorporação 
da biomassa dos adubos verdes e das plantas espontâneas ao solo ou a deposição da biomassa na superfície do solo, ou seja, com ou sem revolvimento do solo) produzida pelos três tratamentos de adubos verdes que constituem as subparcelas. Os tratamentos de adubos verdes corresponderam a duas misturas de espécies (denominadas de coquetéis) e um terceiro tratamento onde é permitido o crescimento de plantas espontâneas.

O coquetel 1 correspondeu a $25 \%$ de gramíneas / oleaginosas $+75 \%$ de leguminosas e o coquetel 2 a $25 \%$ de leguminosas $+75 \%$ de gramíneas / oleaginosas, para ambas áreas de cultivo, o terceiro tratamento consistiu de vegetação espontânea, e todos os tratamentos foram submetidos aos dois manejos (com e sem revolvimento do solo).

As espécies leguminosas utilizadas nos coquetéis são: duas Crotalárias (Crotalaria juncea L. e C. spectabilis Roth), Feijão de Porco (Canavalia ensiformis (L.) DC.), Lablab (Dolichos lablab L.) e duas espécies de Mucuna (Mucuna aterrima (Piper \& Tracy) Holland e M. nivea (Roxb.) DC. ex Wight \& Arn.). As oleaginosas são Girassol (Hellianthus annuus L.) e Mamona (Ricinus communis L.), e Milheto (Pennisetum glaucum (L.) R. Brown), Milho (Zea mays L.) e Sorgo (Sorghum bicolor (L.) Moench) são as espécies do grupo das gramíneas.

Todas as espécies dos coquetéis foram semeadas em 12 linhas espaçadas de $50 \mathrm{~cm}$, em parcelas de $6 \mathrm{~m} \mathrm{x} 8 \mathrm{~m}$, na área de cultivo do melão. Tanto os coquetéis como o melão recebem irrigação por gotejamento (fitas gotejadoras espaçadas a cada $1 \mathrm{~m}$ e com $0,5 \mathrm{~m}$ entre gotejadores). $\mathrm{O}$ corte das plantas de cobertura foi realizado, aproximadamente, aos 70 dias após o plantio.

Para a estimativa da FBN, foram realizadas coletas do material vegetal dos adubos verdes na época do corte, em quadrados de $0,25 \mathrm{~m}^{2}$, lançados aleatoriamente, nas linhas dos coquetéis vegetais, com três repetições por parcela. Nas parcelas correspondentes aos coquetéis, a biomassa de cada uma das espécies semeadas foi pesada separadamente. Para as plantas espontâneas foram determinados o peso total do material composto. Na medida do possível, foram realizadas as identificações das espécies espontâneas e algumas, mais frequentes em todas as parcelas, foram coletadas para serem utilizadas como plantas referência na estimativa da FBN.

Amostras dessas biomassas foram secas em estufa, para determinação da biomassa seca, e preparada para determinação dos teores de $\mathrm{C}(\%), \mathrm{N}$ total $(\%),{ }^{15} \mathrm{~N}(\%)$ e ${ }^{13} \mathrm{C}(\%)$, por espectrometria de massa. Para estimar a FBN será utilizado o método da abundância natural do ${ }^{15} \mathrm{~N}$. $\mathrm{O}$ percentual de nitrogênio da planta derivado do ar (\% Ndda) será calculado utilizando a fórmula descrita por Shearer e Kohl (1986). 
Os conteúdos de $\mathrm{N}$ e C na parte aérea das plantas serão estimados multiplicando os teores dos elementos pelas biomassas correspondentes. A quantidade de $\mathrm{N}$ fixado, na parte aérea das plantas, será estimada multiplicando o valor de \% Ndda pelo conteúdo de $\mathrm{N}$ de cada planta testada, em cada um dos tratamentos.

\section{Resultados e Discussão}

Os dados de biomassa extraídos do cultivo dos coquetéis vegetais contribuíram com maior acúmulo de biomassa quando comparado com os tratamentos onde foi permitido o crescimento das espécies espontâneas (Tabelas 1, 3, 4 e 5).

Entre as leguminosas, a mucuna destacou-se na produção de biomassa, variando de 2,143 a 3,619 t ha ${ }^{-1}$. Em relação às espécies não leguminosas, o milho teve destaque na produção de biomassa (Tabela 1).

Os valores de $\delta{ }^{15} \mathrm{~N}$ em todas as leguminosas estudadas foram menores em relação ao valor médio encontrado pelo girassol e mamona, espécies utilizadas como referência (com a diferença chegando a 7,42\%o), indicando uma considerável absorção de $\mathrm{N}$ atmosférico, possibilitando estimativas confiáveis de $\mathrm{N}$ fixado (Högberg, 1997). Para o sorgo, encontrou-se a o sinal de $\delta{ }^{15} \mathrm{~N}$ diferença de 4,1\%o, em um dos tratamentos (Tabela 2).

Entre as leguminosas utilizadas nos coquetéis vegetais, o lablab e a mucuna foram as espécies que apresentaram menor sinal de ${ }^{15} \mathrm{~N}$. No entanto, todas as leguminosas utilizadas no experimento apresentam-se como fixadoras de N, por apresentarem valores baixos de $\delta{ }^{15} \mathrm{~N}$, quando comparadas com os valores encontrados pelas plantas referência, diferenças que ultrapassam 2 \%o (Tabela 2). 
Tabela 1. Biomassa seca, $\mathrm{C}$ fixado, relação $\mathrm{C} / \mathrm{N}, \mathrm{N}$ acumulado de espécies de diferentes coquetéis vegetais cultivados em pré- plantio do melão irrigado, no semiárido de Pernambuco

\begin{tabular}{|c|c|c|c|c|c|c|c|c|}
\hline & \multicolumn{2}{|c|}{$\begin{array}{c}\text { Biomassa seca (Kg } \\
\left.\text { ha- }^{-1}\right)\end{array}$} & \multicolumn{2}{|c|}{$\underset{\left.\text { ha }^{-1}\right)}{\text { C fixado }}(\mathrm{Kg}$} & \multicolumn{2}{|c|}{ Relação C/N } & \multicolumn{2}{|c|}{$\begin{array}{c}\mathrm{N} \text { acumulado (Kg } \\
\left.\mathbf{h a}^{-1}\right)\end{array}$} \\
\hline & SR & $\mathbf{C R}$ & SR & CR & SR & CR & SR & CR \\
\hline \multicolumn{9}{|c|}{$\begin{array}{l}\text { Coquetel } 1(25 \% \text { GO + } 75 \\
\% \text { L) }\end{array}$} \\
\hline Crotalárias & 269,68 & 636,25 & 114,69 & 265,26 & 30,51 & 23,90 & 4,36 & 10,92 \\
\hline Feijão de porco & 218,90 & 153,30 & 86,33 & 57,37 & 12,51 & 20,89 & 6,77 & 4,16 \\
\hline Lablab & 484,40 & 826,13 & 203,50 & 332,70 & 15,29 & 12,17 & 13,26 & 28,63 \\
\hline Mucunas & 3619,38 & 3361,65 & 1547,55 & 1379,00 & 16,86 & 12,47 & 95,99 & 117,06 \\
\hline Milheto & 26,70 & 86,65 & 10,62 & 32,01 & 21,35 & 23,76 & 0,50 & 1,31 \\
\hline Milho & 2408,30 & 5596,65 & 975,69 & 2195,94 & 31,41 & 51,93 & 35,40 & 47,25 \\
\hline Sorgo & 120,00 & 80,00 & 49,68 & 31,62 & 42,19 & 35,70 & 1,18 & 1,06 \\
\hline Girassol & 429,80 & 633,75 & 173,67 & 225,01 & 30,17 & 28,25 & 10,45 & 8,76 \\
\hline Mamona & 206,55 & 776,30 & 84,59 & 301,36 & 18,13 & 20,89 & 4,93 & 22,83 \\
\hline \multicolumn{9}{|c|}{$\begin{array}{l}\text { Coquetel } 2(25 \% \text { L }+75 \% \\
\text { GO) }\end{array}$} \\
\hline Crotalárias & 429,80 & 573,18 & 173,67 & 233,49 & 30,17 & 18,24 & 10,45 & 15,50 \\
\hline Feijão de porco & 353,13 & 399,40 & 138,74 & 154,25 & 14,17 & 12,46 & 9,51 & 12,45 \\
\hline Lablab & 612,83 & 500,40 & 256,92 & 205,53 & 15,37 & 13,08 & 19,22 & 16,21 \\
\hline Mucunas & 2143,68 & 2473,30 & 922,60 & 1056,34 & 15,16 & 12,30 & 70,71 & 85,95 \\
\hline Milheto & 103,33 & 66,70 & 40,60 & 25,46 & 28,62 & 26,05 & 1,45 & 0,94 \\
\hline Milho & 1182,50 & 3744,45 & 479,46 & 1497,12 & 37,81 & 38,90 & 12,65 & 39,95 \\
\hline Sorgo & 125,70 & 173,30 & 479,46 & 67,44 & 29,03 & 34,27 & 1,69 & 1,97 \\
\hline Girassol & 633,45 & 880,50 & 249,22 & 335,84 & 27,98 & 25,66 & 9,61 & 17,55 \\
\hline Mamona & 463,58 & 656,50 & 183,39 & 255,84 & 28,95 & 22,77 & 6,68 & 10,07 \\
\hline $\begin{array}{l}* \mathrm{GO}=\text { Gramíne } \\
\text { oleaginosas } \\
* \mathrm{~L}=\text { Leguminos }\end{array}$ & & & & & & & & \\
\hline
\end{tabular}

Tabela 2. Teores de $\delta 15 \mathrm{~N} \%$, \%Ndda e $\mathrm{N}$ fixado (Kg ha-1) de espécies de diferentes coquetéis vegetais cultivados em pré-plantio do melão irrigado, no semiárido de Pernambuco. 


\begin{tabular}{|c|c|c|c|c|c|c|c|}
\hline & \multicolumn{2}{|c|}{$\delta^{15} \mathrm{~N} \%$} & \multicolumn{2}{|c|}{$\%$ Ndda } & \multicolumn{2}{|c|}{$\mathrm{N}$ fixado $\left(\mathrm{Kg} \mathrm{ha}^{-1}\right)$} & \multirow{3}{*}{ Tabel } \\
\hline & SR & $\mathbf{C R}$ & SR & $\mathbf{C R}$ & SR & $\mathbf{C R}$ & \\
\hline \multicolumn{7}{|c|}{ Coquetel $1(25 \%$ GO + $75 \%$ L) } & \\
\hline Crotalárias & 0,84 & 0,59 & 72,59 & 77,43 & 3,17 & 8,33 & a 3. \\
\hline Feijão de porco & 1,01 & 2,31 & 70,91 & 54,84 & 4,45 & 2,81 & \multirow[t]{2}{*}{ Teore } \\
\hline Lablab & $-0,30$ & 0,06 & 88,64 & 84,55 & 11,62 & 24,22 & \\
\hline Mucunas & $-0,22$ & 0,34 & 79,35 & 73,47 & 76,17 & 85,46 & s de \\
\hline Milheto & 5,56 & 5,69 & 6,17 & 10,17 & 0,03 & 0,12 & \multirow{3}{*}{$\begin{array}{l}\text { Biom } \\
\text { assa }\end{array}$} \\
\hline Milho & 3,90 & 4,90 & 34,15 & $-21,36$ & 12,66 & $-13,54$ & \\
\hline Sorgo & 1,82 & 4,07 & 69,29 & 35,70 & 0,82 & 0,38 & \\
\hline Girassol & 5,03 & 6,89 & 12,92 & $-7,69$ & 2,22 & $-0,64$ & \multirow{3}{*}{$\begin{array}{l}\text { seca, } \\
\text { C } \\
\text { fixad }\end{array}$} \\
\hline Mamona & 6,81 & 5,77 & $-12,88$ & 7,66 & $-1,23$ & $-2,95$ & \\
\hline \multicolumn{7}{|c|}{ Coquetel $2(25 \%$ L $+75 \%$ GO $)$} & \\
\hline Crotalárias & 0,09 & 0,30 & 12,92 & 82,20 & 2,22 & 12,88 & \multirow{3}{*}{$\begin{array}{l}\mathrm{o} \\
\text { Relaç }\end{array}$} \\
\hline Feijão de porco & 0,43 & 1,21 & 80,55 & 71,15 & 7,36 & 9,78 & \\
\hline Lablab & $-0,45$ & $-0,16$ & 91,37 & 88,03 & 17,35 & 14,31 & \\
\hline Mucunas & 0,56 & $-0,77$ & 70,80 & 87,66 & 48,02 & 75,33 & \multirow{3}{*}{$\begin{array}{l}\text { ão } \\
\mathrm{C} / \mathrm{N}\end{array}$} \\
\hline Milheto & 6,09 & 6,37 & 3,75 & 4,23 & $-0,08$ & 0,01 & \\
\hline Milho & 5,22 & 5,79 & 17,49 & 13,01 & 1,65 & 3,66 & \\
\hline Sorgo & 4,32 & 5,89 & 31,80 & 9,88 & 0,54 & 0,19 & \multirow{3}{*}{$\begin{array}{l}\mathrm{N} \\
\text { acum }\end{array}$} \\
\hline Girassol & 6,15 & 7,09 & 2,49 & $-5,78$ & $-0,20$ & 0,15 & \\
\hline Mamona & 6,52 & 6,22 & $-2,62$ & 5,58 & $-0,54$ & 0,92 & \\
\hline \multicolumn{7}{|c|}{ * GO = Gramíneas e oleaginosas e L= Leguminosas } & lado \\
\hline$* \mathrm{SR}=$ sem revo & olvimento & & & & & & de \\
\hline
\end{tabular}

espécies de diferentes plantas espontâneas que cresceram no plot dos meloteiros, no semiárido de Pernambuco.

\begin{tabular}{|c|c|c|c|c|c|c|c|c|}
\hline \multirow{2}{*}{$\begin{array}{l}\text { Plantas espontâneas } \\
\text { Leguminosas } \\
\end{array}$} & \multicolumn{2}{|c|}{ Biomass a seca $\left(\mathrm{Kg} \mathrm{ha}{ }^{-1}\right)$} & \multicolumn{2}{|c|}{$\mathrm{C}$ fixado $\left(\mathrm{Kg} \mathrm{ha}^{-1}\right)$} & \multicolumn{2}{|c|}{ Relação $\mathrm{C} / \mathrm{N}$} & \multicolumn{2}{|c|}{ N acumulado $\left(\mathrm{Kg} \mathrm{ha}^{-1}\right)$} \\
\hline & $\$$ & $\mathrm{CR}$ & SR & $\mathrm{CR}$ & SR & $\mathrm{CR}$ & $S R$ & CR \\
\hline Indigofera Hirsuta & 213,33 & 726,83 & 92,83 & 304,08 & 17,43 & 14,20 & 5,30 & 21,72 \\
\hline Macroptilium martii & 447,40 & - & 187,68 & - & 26,19 & - & 8,19 & - \\
\hline M. lathroides & 503,90 & - & 215,47 & - & 20,38 & - & 10,33 & - \\
\hline Mimosa pudica & 53,30 & - & 23,60 & - & 18,80 & - & 1,26 & - \\
\hline Gramíneas & - & - & - & - & & - & & - \\
\hline Digitaria bicomis & 897,83 & 1666,53 & 355,54 & 643,36 & 51,01 & 44,06 & 6,87 & 15,30 \\
\hline Cenchrus echinatus & - & 226,65 & - & 89,33 & - & 42,62 & - & 2,00 \\
\hline Demais familias & 886,65 & 444,47 & 332,11 & 149,40 & 31,96 & 32,03 & 10,69 & 4,50 \\
\hline
\end{tabular}

\section{Conclusões}

O cultivo e adubação verde com coquetéis vegetais previamente ao cultivo do melão irrigado pode ser uma boa alternativa com uma considerável adição de biomassa e $\mathrm{C}$ e $\mathrm{N}$ ao sistema em relação à presença apenas de plantas espontâneas. Destacando assim, que existe um maior acúmulo $\mathrm{kg} \mathrm{ha}^{-1}$ de $\mathrm{C}$ e de $\mathrm{N}$ com relação às espontâneas, valores esses referenciados nos resultados. 
A partir dos resultados obtidos, pode-se observar que o lablab é a planta com maior potencial de fixação biológica do N. No entanto, as mucunas foram as plantas que apresentaram maior contribuição de $\mathrm{N}$ ao sistema via FBN, por apresentar maior produção de biomassa.

Sendo assim, as mucunas apresentam-se como boa alternativa de cultivo como planta fixadora de $\mathrm{N}$ e fornecedora de biomassa como adubo verde.

\section{Referências}

ASHRAF, A.; MAHMOOD, T.; AZAM, F.; QURESHI, R. M. Comparative effects of applying leguminous and non-leguminous green manures and inorganic $\mathrm{N}$ on biomass yield and nitrogen uptake in flooded rice (Oryza sativa L.). Biology and Fertility of Soils, v.40, p.147-152, 2004.

BARRETO, A. C.; FERNANDES, M. F. Recomendações técnicas para o uso da adubação verde em solos de tabuleiros costeiros. Aracaju: Embrapa Tabuleiros Costeiros, 2001, 24p.

DIAS, P. F.; SOUTO, S. M.; RESENDE, A. S.; URQUIAGA, S.; ROCHA, G. P.; MOREIRA, J.F.; FRANCO, A. A. Transferência do N fixado por leguminosas arbóreas para o capim Survenola crescido em consórcio. Ciência Rural, v.37, p.352-356, 2007.

FERREIRA NETO, Reginaldo Alves. Nitrogênio fixado em cultivo de melão sob adubação verde no município de Juazeiro, Bahia. 2013.

GRAHAM, P. H.; VANCE, C. P. Legumes: importance and constraints to greater utilization. Plant and Soil, v.131, n.3, p.872-877, 2003.

HÖGBERG, Peter. Tansley Review No. $9515 \mathrm{~N}$ natural abundance in soil-plant systems. New Phytologist, v. 137, n. 2, p. 179-203, 1997.

IBGE. Semiárido. Disponível em: <http://www.ibge.gov.br/home/geociencias/geografia/ semiarido.shtm?c=4.>. Acesso em: 17 ago 2016.

IBGE. Produção agrícola municipal 2013. Disponível em: <ftp://ftp.ibge.gov.br/ProducaoAgricola/ProduçãoAgricola_Municipal_\%5Banual\%5D/2011/tabela s_pdf/tabela04.pdf>. Acesso em: 13 dez.2014.

OJIEM J.O.; FRANKE A.C.; VANLAUWE B.; DE RIDDER N.; GILLER K.E. Benefits of legume-maize rotations: Assessing the impact of diversity on the productivity of smallholders in Western Kenya. Field Crops Research, v.168, p. 75-85, 2014.

PAULINO, G.M; ALVES, B.J.R; BARROSO, D.G; URQUIAGA, S.; ESPINDOLA J.A.A. Fixação biológica e transferência de nitrogênio por leguminosas em pomar orgânico de mangueira e gravioleira. Pesquisa Agropecuária Brasileira, v.44, n.12, p.1598-1607, 2009. 
PERIN, A.; GUERRA, J. G. M.; ESPINDOLA, J. A. A.; TEIXEIRA, M. G.; BUSQUET, R. N. B. Desempenho de bananeiras consorciadas com leguminosas herbáceas perenes. Ciência e Agrotecnologia, v. 33, n. 6, p. 1511-1517, 2009.

SHEARER, G.; KOHL, D.H. N2 fixation in field settings: estimations based on natural 15N abundance. Australian Journal of Plant Physiology, v.13, p.699-756, 1986.

SUDENE. Semiárido. Disponível em:<http://www.sudene.gov.br/acessoainforma\% C3\% A7\% C3\%A3o/institucional/ area-de-atuacao-da-sudene/semiarido> Acesso em: 17 ago 2016.

TORRES, J.L.R.; PEREIRA M.G.; ANDRIOLI, I.; POLIDORO, J.C. \& FABIAN, A.J.; Decomposição e liberação de nitrogênio de resíduos culturais de plantas de cobertura em um solo de Cerrado. Revista Brasileira de Ciência do Solo, 29:609-618, 2005.

ZOTARELLI, L.; ALVES, B. J. R.; URQUIAGA, S.; BODDEY, R. M.; SIX, J. Impact of tillage and crop rotation on light fraction and intra-aggregate soil organic matter in two oxisols. Soil \& Tillage Research, Amsterdam, v. 95, n. 1-2, p. 196-206, 2007. 